Journal of Animal and Veterinary Advances 10 (15): 2010-2015, 2011

ISSN: $1680-5593$

(C) Medwell Journals, 2011

\title{
Comparative Analysis of 185 and 285 rDNA Sequences of Schistosoma japonicum from Mainland China, the Philippines and Japan
}

\author{
${ }^{1,2}$ F. Chen, ${ }^{1} \mathrm{~J}$. Li, ${ }^{3} \mathrm{H}$. Sugiyama, ${ }^{1}$ Y.B. Weng, ${ }^{4} \mathrm{~F}$.C. Zou, ${ }^{1}$ R.Q. Lin, ${ }^{1}$ Z.G. Yuan, \\ ${ }^{5}$ H.Q. Song, ${ }^{4,} \mathrm{X}$.Q. Zhu and ${ }^{2}$ G.H. Zhao \\ ${ }^{1}$ College of Veterinary Medicine, South China Agricultural University, \\ 510642 Guangzhou, Guangdong Province, China \\ ${ }^{2}$ College of Veterinary Medicine, Northwest A and F University, \\ 712100 Yangling, Shanxi Province, China \\ ${ }^{3}$ Department of Parasitology, National Institute of Infectious Diseases, \\ 113-8421 Tokyo, Japan \\ ${ }^{4}$ College of Animal Science and Technology, Yunnan Agricultural University, \\ 650201 Kunming, Yunnan Province, China \\ ${ }^{5}$ State Key Laboratory of Veterinary Etiological Biology, \\ Key Laboratory of Veterinary Parasitology of Gansu Province, \\ Lanzhou Veterinary Research Institute, CAAS, 730046 Lanzhou, Gansu Province, China
}

\begin{abstract}
In the present study, a portion of the 18S and 28S ribosomal DNA (rDNA) sequences of 35 Schistosoma japonicum isolates representing three geographical strains from mainland China, the Philippines and Japan were amplified and compared and phylogenetic relationships were also reconstructed by Unweighted Pair-Group Method with Arithmetic averages (UPGMA) using combined 18S and 28S rDNA sequences as well as the corresponding sequences of other species belonging to the Schistosoma genus available in the public database. The results indicated that the partial $18 \mathrm{~S}$ and $28 \mathrm{~S}$ rDNA sequences of all S.japonicum isolates were 745 and 618 bp, respectively and displayed low genetic variation among S. japonicum strains and isolates. Phylogenetic analysis revealed that the combined 18S and 28S rDNA sequences were not able to distinguish $S$. japonicum isolates from three geographical origins but provided an effective molecular marker for the inter-species phylogenetic analysis and differential identification of different Schistosoma species.
\end{abstract}

Key words: Schistosoma japonicum, 18S rDNA, 28S rDNA, phylogenetic analysis, mainland China, Philippines, Japan

\section{INTRODUCTION}

Schistosomiasis caused by trematodes of the genus Schistosoma is a parasitic disease of medical and veterinary importance in a number of countries with an estimated approximately 200 million people being infected and approximately 200,000 deaths per year (Chitsulo et al., 2004; Taylor, 2008; Huyse et al., 2009). Among six human schistosomes (Schistosoma japonicum, Schistosoma haematobium, Schistosoma mansoni (Schistosoma intercalatum $=S$. guineensis), Schistosoma mekongi and Schistosoma malayensis), three main species (S. japonicum, S. haematobium and S. mansoni) are reported to have significant human health and socialeconomic impact (Zhao et al., 2009a). Schistosomiasis japonica, one of the most serious schistosomiasis caused by $S$. japonicum was mainly distributed in China, Japan, the Philippines and parts of Indonesia and caused various degrees of morbidity and mortality (Zhou et al., 2005; Zhao et al., 2009a, b). In addition to the six human schistosomes, other 13 Schistosoma species have been identified as parasites in animals. $S$. hippopotami and $S$. edwardiense are found in hippopotamus (Morgan et al., 2003), S. mattheei, S. bovis, S. leiperi, S. margrebowiei, $S$. curassoni, $S$. indicum, $S$. spindale and $S$. nasale found in ruminant animals such as cattle, buffalo, sheep, goat and lechwe (De Bont et al., 1994; Vercruysse et al., 2003; Singh et al., 2004; Vercruysse and Gabriel, 2005; Littlewood et al., 2006; Sato et al., 2008) and S. rodhaini, S. incognitum, S. sinensium mainly inhabited in rodents and carnivores (Bunnag et al., 1983; Walker et al., 1989; Zhang et al., 2001).

Corresponding Author: G.H. Zhao, Department of Veterinary Immunology, College of Veterinary Medicine, Northwest A and F University, 712100 Yangling, Shaanxi Province, The People's Republic of China 
Ribosomal DNA (rDNA) forms a tandem array of repeat sequences and each repeat includes $18 \mathrm{~S}, 5.8 \mathrm{~S}$ and 28S subunits separated by spacers (Zhao et al., 2011). The $18 \mathrm{~S}$ and $28 \mathrm{~S}$ rDNA sequences have been identified as good genetic markers for identification and phylogenetic studies of members of the genus Schistosoma (Johnston et al., 1993; Barker and Blair, 1996; Attwood et al., 2002; Lockyer et al., 2003; Webster et al., 2006). Recent studies also have demonstrated that different $S$. japonicum strains from mainland China and the Philippines has extensive homology in 18S rDNA sequences and 28S rDNA-D2 domain was also relatively stable among species (Yu et al., 2000; Li et al., 2008). But sequence variation in 18 and 28S rDNA among S. japonicum strains from mainland China, the Philippines and Japan have not been investigated comprehensively.

The objectives of the present study were to examine sequence variation in the $18 \mathrm{~S}$ and $28 \mathrm{~S} \mathrm{rDNA}$ among S. japonicum isolates from different endemic regions in mainland China, the Philippines and Japan and to reconstruct the phylogenetic relationships among members of the Schistosoma genus using the combined partial $18 \mathrm{~S}$ and $28 \mathrm{~S}$ rDNA sequences.

\section{MATERIALS AND METHODS}

Parasites and isolation of genomic DNA: The 35 $S$. japonicum isolates were collected from the endemic areas in mainland China, the Philippines and Japan with sample codes, geographical origin and gender shown in Table 1. The male and female adult parasites were fixed in $70 \%$ molecular grade ethanol and stored at $-20^{\circ} \mathrm{C}$ before extraction of genomic DNA. Total genomic DNA was extracted from individual parasites by SDS/proteinase K treatment, column-purified (Wizard ${ }^{\circledR}$ SV Genomic DNA Purification System, Promega) and eluted into $60 \mu \mathrm{L} \mathrm{H}_{2} \mathrm{O}$ according to the manufacturer's recommendations.

Enzymatic amplification and sequencing: A portion of the $18 \mathrm{~S}$ (p18S) and 28S rDNA were amplified with primers $18 \mathrm{Su}$ and $18 \mathrm{Sd}, 28 \mathrm{Su}$ and $28 \mathrm{Sd}$, respectively (Table 2 ). PCR reactions ( $25 \mu \mathrm{L}$ ) were performed in $2 \mathrm{mM}$ of $\mathrm{MgCl}_{2}$, $2.5 \mu \mathrm{M}$ of each primer, $2.5 \mu \mathrm{L} 10 \times$ rTaq buffer, $0.2 \mathrm{mM}$ of each dNTPs, $1.25 \mathrm{U}$ of rTaq DNA polymerase (Takara) and $1 \mu \mathrm{L}$ of DNA sample in a thermocycler (Biometra) under the following conditions: after an initial denaturation at $94^{\circ} \mathrm{C}$ for $5 \mathrm{~min}$ then $94^{\circ} \mathrm{C}$ for $1 \mathrm{~min}$ (denaturation); $45^{\circ} \mathrm{C}$ for $30 \mathrm{sec}$ (annealing); $72^{\circ} \mathrm{C}$ for $1 \mathrm{~min}$ (extension) for 35 cycles followed by a final extension at $72^{\circ} \mathrm{C}$ for $10 \mathrm{~min}$. These optimized cycling conditions for the specific and efficient amplification of both rDNA fragments were obtained after adjusting annealing temperatures. Each amplicon $(4 \mu \mathrm{L})$ was examined by agarose $(1 \%)$ gel electrophoresis to validate amplification efficiency. The p18S and p28S amplicons of 35 samples were sequenced by BGI-Guangzhou company from both directions using the same primers as used in primary amplification.

Sequences alignment and analysis: Sequences of the p18S and p28S rDNA were separately aligned using the computer program Clustal X 1.81 (Thompson et al., 1997). Meanwhile, Megalign procedure within the DNAStar 5.0 (Burland, 2000) was also used to analyze sequence similarity and to calculate transition and transversion.

Table 1: Information of Schistosoma samples used in the present study

\begin{tabular}{|c|c|c|c|c|c|}
\hline \multirow[b]{2}{*}{ Species/sample codes } & \multirow[b]{2}{*}{ Geographical strains } & \multirow[b]{2}{*}{ Geographical origin } & \multirow[b]{2}{*}{ Gender } & \multicolumn{2}{|c|}{ GenBank accession number } \\
\hline & & & & p18S rDNA & $\mathrm{p} 28 \mathrm{~S}$ rDNA \\
\hline Schistosoma joponicum/SjYeF55 & Mainland China & Yunnan (Eryuan) & Femal & $\mathrm{JF} 721330$ & $\mathrm{JF} 721363$ \\
\hline S. joponicum/SjYeM55 & Mainland China & Yunnan (Eryuan) & Male & JF721331 & $\mathrm{JF} 721364$ \\
\hline S. joponicum/SjHyF54 & Mainland China & Hunan (Yueyang) & Femal & $\mathrm{JF} 721328$ & $\mathrm{JF} 721361$ \\
\hline S. joponicum/SjHyM54 & Mainland China & Hunan (Yueyang) & Male & $\mathrm{JF} 721329$ & $\mathrm{JF} 721362$ \\
\hline S. joponicum $/ \mathrm{SjZjF} 60$ & Mainland China & Zhejiang & Femal & $\mathrm{JF} 721326$ & $\mathrm{JF} 721365$ \\
\hline S. joponicum/SjZjM60 & Mainland China & Zhejiang & Male & $\mathrm{JF} 721327$ & $\mathrm{JF} 721366$ \\
\hline S. joponicum/SjLeF1 & The Philippines & Letye & Femal & $\mathrm{JF} 721338$ & $\mathrm{JF} 721373$ \\
\hline S. joponicum/SjLeF2 & The Philippines & Letye & Femal & $\mathrm{JF} 721339$ & $\mathrm{JF} 721374$ \\
\hline S. joponicum $/ \mathrm{SjLeF} 4$ & The Philippines & Letye & Femal & $\mathrm{JF} 721340$ & $\mathrm{JF} 721375$ \\
\hline S. joponicum/SjLeM1 & The Philippines & Letye & Male & $\mathrm{JF} 721341$ & $\mathrm{JF} 721376$ \\
\hline S. joponicum/SjLeM2 & The Philippines & Letye & Male & $\mathrm{JF} 721342$ & $\mathrm{JF} 721377$ \\
\hline S. joponicum/SjLeM4 & The Philippines & Letye & Male & $\mathrm{JF} 721343$ & $\mathrm{JF} 721378$ \\
\hline S. joponicum/SjMiF1 & The Philippines & Mindoro & Femal & JF721349 & $\mathrm{JF} 721384$ \\
\hline S. joponicum/SjMiF2 & The Philipp ines & Mindoro & Femal & $\mathrm{JF} 721350$ & $\mathrm{JF} 721385$ \\
\hline S. joponicum/SjMiF4 & The Philippines & Mindoro & Femal & $\mathrm{JF} 721351$ & $\mathrm{JF} 721386$ \\
\hline S. joponicum/SjMiM1 & The Philippines & Mindoro & Male & $\mathrm{JF} 721352$ & $\mathrm{JF} 721387$ \\
\hline S. joponicum/SjMiM2 & The Philippines & Mindoro & Male & $\mathrm{JF} 721353$ & $\mathrm{JF} 721388$ \\
\hline S. joponicum/SjMiM3 & The Philippines & Mindoro & Male & $\mathrm{JF} 721354$ & JF721389 \\
\hline
\end{tabular}


Table 1: Continue

\begin{tabular}{|c|c|c|c|c|c|}
\hline \multirow[b]{2}{*}{ Species/sample codes } & \multirow[b]{2}{*}{ Geographical strains } & \multirow[b]{2}{*}{ Geographical origin } & \multirow[b]{2}{*}{ Gender } & \multicolumn{2}{|c|}{ GenBank accession number } \\
\hline & & & & p18S rDNA & p $28 \mathrm{~S}$ rDNA \\
\hline S. joponicum $/ \mathrm{SjSoF} 2$ & The Philippines & Sorsogor & Femal & JF721344 & JF721379 \\
\hline S. joponicum $/ \mathrm{SjSoF} 3$ & The Philippines & Sorsogor & Femal & $\mathrm{JF} 721345$ & JF721380 \\
\hline S. joponicum $/ \mathrm{SjSoM} 1$ & The Philippines & Sorsogor & Male & $\mathrm{JF} 721346$ & JF721381 \\
\hline S. joponicum $/ \mathrm{SjSoM} 3$ & The Philippines & Sorsogor & Male & JF721347 & JF721382 \\
\hline S. joponicum/SjSoM4 & The Philippines & Sorsogor & Male & $\mathrm{JF} 721348$ & JF721383 \\
\hline S. joponicum $/ \mathrm{SjAsF1}$ & The Philippines & Asuncium & Femal & $\mathrm{JF} 721332$ & $\mathrm{JF} 721367$ \\
\hline S. joponicum/SjAsF3 & The Philippines & Asuncium & Femal & JF721333 & JF721368 \\
\hline S. joponicum $/ \mathrm{SjAsF} 4$ & The Philippines & Asuncium & Femal & JF721334 & JF721369 \\
\hline S. joponicum/SjAsM1 & The Philippines & Asuncium & Male & $\mathrm{JF} 721335$ & $\mathrm{JF} 721370$ \\
\hline S. joponicum/SjAsM2 & The Philippines & Asuncium & Male & JF721336 & JF721371 \\
\hline S. joponicum/SjAsM4 & The Philippines & Asuncium & Male & JF721337 & $\mathrm{JF} 721372$ \\
\hline S. joponicum/SjYYF1 & Japan & Yamanashi & Femal & JF721355 & JF721390 \\
\hline S. joponicum/SjYYF2 & Japan & Yamanashi & Femal & JF721356 & JF721391 \\
\hline S. joponicum/SjYYF4 & Japan & Yamanashi & Femal & JF721357 & JF721392 \\
\hline S. joponicum/SjYYM2 & Japan & Yamanashi & Male & JF721358 & JF721393 \\
\hline S. joponicum/SjYYM3 & Japan & Yamanashi & Male & JF721359 & JF721394 \\
\hline S. joponicum/SjYYM4 & Japan & Yamanashi & Male & $\mathrm{JF} 721360$ & JF721395 \\
\hline S. intercalatum & - & - & - & AY157235 & AY157262 \\
\hline S. rodhaini & - & - & - & AY157230 & AY157256 \\
\hline S. spindale & - & - & - & Z11979 & Z46505 \\
\hline S. bovis - & - & - & AY157238 & AY157266 & \\
\hline S. edwardiense & - & - & - & AY197344 & AY197344 \\
\hline S. haematobium & - & - & - & Z11976 & Z46521 \\
\hline S. hippopotom & - & - & - & AY197343 & AY197343 \\
\hline S. incognitum & - & - & - & AY157229 & AY157255 \\
\hline S. joponicum & - & - & - & AY157226 & Z46504 \\
\hline S. leiperi - & - & - & AY157234 & AY157261 & \\
\hline S. malayensis & - & - & - & AY157227 & AY157252 \\
\hline S. mattheei & - & - & - & AY157237 & AY157265 \\
\hline S. mekongi - & - & - & AY157228 & AY157253 & \\
\hline S. nasale - & - & - & AY 157232 & AY157259 & \\
\hline S. sinensium & - & - & - & AY157225 & AY157251 \\
\hline S. mansoni & - & - & - & M62652 & Z46503 \\
\hline S. indicum - & - & - & AY157231 & AY157258 & \\
\hline
\end{tabular}

Table 2: Sequences of primers used to amplify a portion of the $18 \mathrm{~S}$ and $28 \mathrm{~S}$ ribosomal DNA from Schistosoma joponicum isolates from mainland China, the Philippines and Japan

\begin{tabular}{lll}
\hline Name of primer & \multicolumn{1}{c}{ Sequence (5'-3') } & Product length \\
\hline For 18S rDNA & - & $745 \mathrm{bp}$ \\
18Su (forward) & CTTATGCTGTGCCTGTTACATT & - \\
18Sd (reverse) & TTACTTCGGATCCGAAAACCAAC & - \\
For 28S rDNA & - & $618 \mathrm{bp}$ \\
28Su1 (forward) & GGGTATGTGTAGACGTTCTTAT & - \\
28Sd (reverse) & AACACAAGGTCGCATGTCTACGT & - \\
\hline
\end{tabular}

These sequences were used for phylogenetic analyses. The sequences of the two $r R N A$ genes were concatenated into single alignments. The Unweighted Pair-Group Method based on Arithmetic averages (UPGMA) (Sneath and Sokal, 1973) in MEGA v. 4.0 (Tamura et al., 2007) was carried out to examine the genetic relationship, starting from a distance matrix based on the Kimura 2-parameter index (Kimura, 1980). The consensus tree was obtained after bootstrap analysis with 1, 000 replications. The pairwise comparisons were made of the level of sequence differences (Chilton et al., 1995). To study the phylogenetic relationships of Schistosoma, the corresponding sequences of other Schistosoma species/isolates namely; $S$. intercalatum, $S$. rodhaini, S. spindale, S. bovis, S. edwardiense, S. haematobium, S. hippopotam, S. incognitum, S. indicum, S. japonicum,
S. leiperi, S. malayensis, S. mattheei, S. mekongi, $S$. nasale, $S$. sinensium and $S$. mansoni obtained from GenBank were also used for phylogenetic analyses. The phylograms were drawn using the Tree View program version 1.65 (Page, 1996).

\section{RESULTS AND DISCUSSION}

Genomic DNA was prepared from 35 individual adult trematodes (including male and female $S$. japonicum) representing three geographical strains from mainland China, the Philippines and Japan. Amplicons of p18S and p28S rDNA $(\sim 750$ and 620 bp, respectively) were amplified. For each rDNA fragment, no size variation was detected on $1 \%$ agarose gel among any of the amplicons examined in dicating that the PCR primers and reactions of this study had good specificity (Fig. 1).

To examine sequence variations in the two rDNA fragments among three geographical strains, amplicons of p18S and p28S rDNA were subjected to direct sequencing. The sequences of p18S and p28S rDNA were 745 and 618 bp in length, respectively. One variable nucleotide position was identified in the sequences of p18S and three in $\mathrm{p} 28 \mathrm{~S}$ rDNA with intra-specific variation of $0.14 \%(1 / 704)$ and $0.51 \%(3 / 586)$ for p18S and $\mathrm{p} 28 \mathrm{~S}$ 


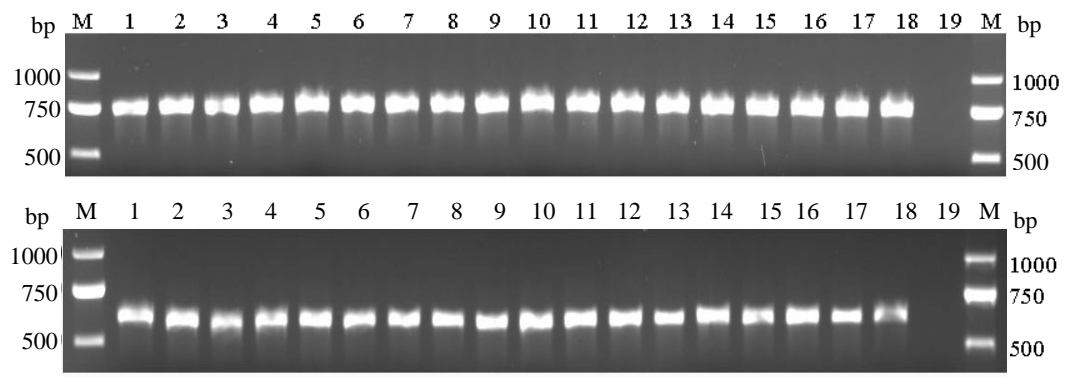

Fig. 1: Representative PCR products for a portion of the 18S (upper) and 28S (bottom) ribosomal DNA of Schistosoma japonicum isolates in mainland China, the Philippines and Japan. Lanes 1-18 represent samples SjYeM55, SjHyM54, SjZjF60, SjLeM1, SjLeM4, SjLeF1, SjMiM1, SjMiF1, SjMiF4, SjSoM3, SjSoM4, SjSoF2, SjAsM1, SjAsM2, SjAsF3, SjYYM2, SjYYM3, SjYYF1, respectively (cf. Table 1). Lane 19 represents no-DNA control. M represents a DNA size marker (ordinate values in bp)

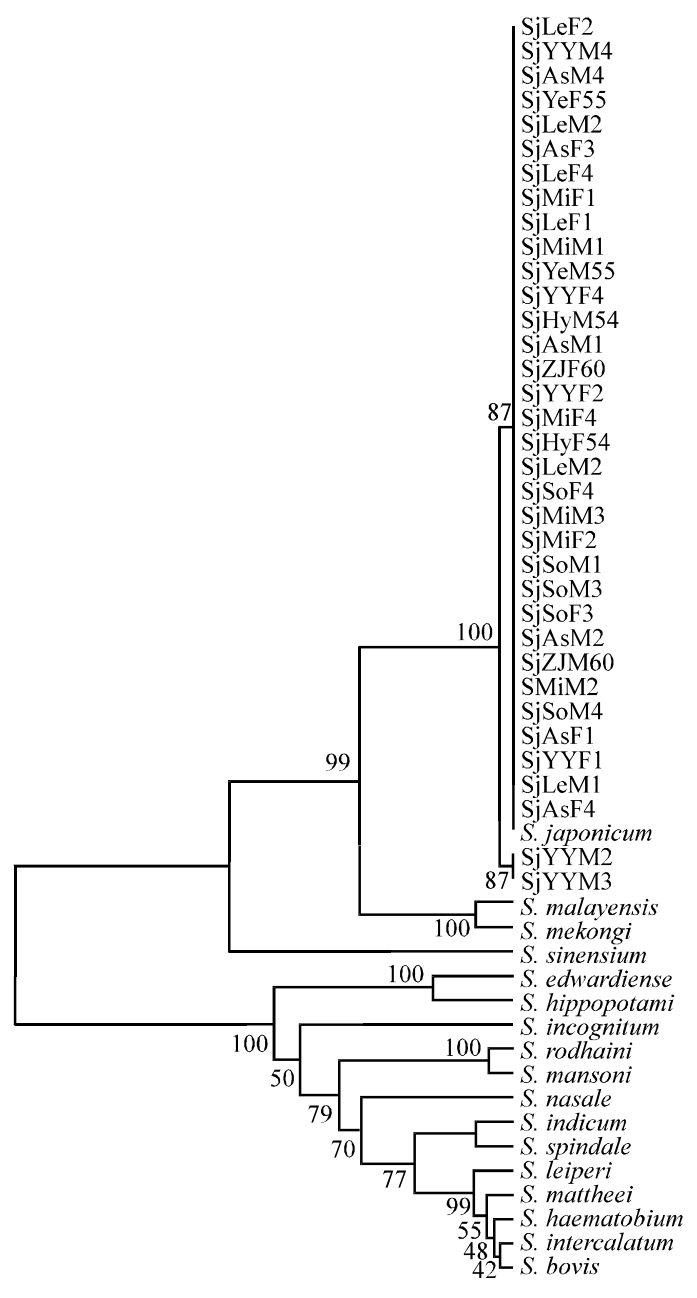

$\begin{array}{lllllll}0.030 & 0.025 & 0.020 & 0.015 & 0.010 & 0.005 & 0.000\end{array}$

Fig. 2: Phylogenetic relationships of Schistosoma species/isolates inferred by UPGMA analysis using the combined sequences of partial 18S and p28S ribosomal DNA. Numbers at nodes indicate bootstrap values (\%) resulting from UPGMA tree
rDNA, respectively. There were one transitions $(A<->G)$ with intra-specific variation for p18S and two $(A<->G)$ and one $(\mathrm{C}<->\mathrm{T})$ transitions for $\mathrm{p} 28 \mathrm{~S}$ rDNA. It appeared that the main transition was $A<->G$ and there was no transversion within these two fragments. The low variations between the $\mathrm{p} 18 \mathrm{~S}$ and $\mathrm{p} 28 \mathrm{~S}$ rDNA were consistent with previous reports ( $\mathrm{Yu}$ et al., 2000; Li et al., 2008).

The combined sequences of $\mathrm{p} 18 \mathrm{~S}$ and $\mathrm{p} 28 \mathrm{~S}$ rDNA were aligned over a consensus length of 1,290 bp. The phylogenetic relationships among the individual $S$. japonicum isolates were constructed by UPGMA analyses (Fig. 2). The phylogenetic tree consisted of two large clades.

All of the S.japonicum isolates from mainland China, the Philippines and Japan clustered in the S. japonicum clade, grouped with $S$. malayensis and $S$. mekongi and sistered to $S$. sinensium. All of the other Schistosoma species grouped in the other large clade with high bootstrap values.

The combined sequences of p18S and p28S rDNA allowed the unequivocal differentiation of all the Schistosoma species examined in the present study which is consistent with previous studies (Johnston et al., 1993; Littlewood and Johnston, 1995; Attwood et al., 2002; Morgan et al., 2003; Webster et al., 2006).

\section{CONCLUSION}

The present study revealed low level variation in p18S and p28S rDNA sequences among 35 S. japonicum isolates from mainland China, the Philippines and Japan. The combined p18S and p28S rDNA sequences were highly conserved and could not distinguish S.japonicum isolates from three geographical origins by phylogenetic analysis but could differentiate species in the Schistosoma genus suggesting that the p18S and p28S rDNA sequences were not suitable markers for studying population relationships among $S$.japonicum isolates but 
is an effective genetic marker for inter-species phylogenetic analysis of Schistosoma and identification of schistosomes.

\section{ACKNOWLEDGEMENTS}

Project support was provided in part by the National Basic Research Program (973 program) of China (Grant No. 2007CB513104), the special funds for talents in Northwest $A$ and F University, the National Natural Science Foundation of China (Grant No. 30960280), the Yunnan Provincial program for Introducing High-level Scientists (Grant No. 2009CI125) and the program for Changjiang scholars and innovative research team in university (Grant No. IRT0723). Professor Baozhen Qian of Bioengineering Institute, Zhejiang Academy of Medical Sciences, China was thanked for providing some $S$. japonicum samples used in the present study.

\section{REFERENCES}

Attwood, S.W., E.S. Upatham, X.H. Meng, D.C. Qiu and V.R. Southgate, 2002. The phylogeography of Asian Schistosoma (Trematoda: Schistosomatidae). Parasitology, 125: 99-112.

Barker, S.C. and D. Blair, 1996. Molecular phylogeny of Schistosoma species supports traditional groupings within the genus. J. Parasitol., 82: 292-298.

Bunnag, T., S. Thirachandra, P. Impand, P. Vorasanta and S. Imlarp, 1983. Schistosoma incognitum and its zoonotic potential role in Phitsanulok and Phichit provinces, northern Thailand. Southeast Asian J. Trop. Med. Public Health, 14: 163-170.

Burland, T.G., 2000. DNASTAR's lasergene sequence analysis software. Methods Mol. Biol., 132: 71-91.

Chilton, N.B., R.B. Gasser and I. Beveridge, 1995. Differences in a ribosomal DNA sequence of morphologically indistinguishable species within the Hypodontus macropi complex (Nematoda: Strongyloidea). Int. J. Parasitol., 25: 647-651.

Chitsulo, L., P. Loverde and D. Engels, 2004. Schistosomiasis. Nat. Rev. Microbiol., 2: 12-13.

De Bont, J., J. Vercruysse, V.R. Southgate, D. Rollinson and A. Kaukas, 1994. Cattle schistosomiasis in Zambia. J. Helminthol., 38: 295-299.

Huyse, T., B.L. Webster, S. Geldof, J.R. Stothard, O.T. Diaw, K. Polman and D. Rollinson, 2009. Bidirectional introgressive hybridization between a cattle and human schistosome species. PLoS. Pathog., 5: e1000571-e1000571.

Johnston, D.A., R.A. Kane and D. Rollinson, 1993. Small subunit (18S) ribosomal RNA gene divergence in the genus Schistosoma. Parasitology, 107: 147-156.
Kimura, M., 1980. A simple method for estimating evolutionary rates of base substitutions through comparative studies of nucleotide sequences. J. Mol. Evol., 16: 111-120.

Li, H.J., Y.S. Liang, J.R. Dai, Y.H. Tao, W. Wang, G.L. Qu and J.Y. Wei, 2008. Homology of $18 \mathrm{~S}$ small subunit ribosomal RNA gene among species and strains of Schistosoma and sensitivity of PCR assay to detect single cercaria. Chin. J. Schisto. Control, 6: 418-422.

Littlewood, D.T. and D.A. Johnston, 1995. Molecular phylogenetics of the four Schistosoma species groups determined with partial $28 \mathrm{~S}$ ribosomal RNA gene sequences. Parasitology, 111: 167-175.

Littlewood, D.T.J., A.E. Lockyer, B.L. Webster, D.A. Johnston and T.H. Le, 2006. The complete mitochondrial genomes of Schistosoma haematobium and Schistosoma spindale and the evolutionary history of mitochondrial genome changes among parasitic flatworms. Mol. Phylogenet. Evol., 39: 452-467.

Lockyer, A.E., P.D. Olson, P. Ostergaard, D. Rollinson and D.A. Johnston et al., 2003. The phylogeny of the Schistosomatidae based on three genes with emphasis on the interrelationships of Schistosoma Weinland, 1858. Parasitology, 126: 203-224.

Morgan, J.A.T., R.J. DeJong, F. Kazibwe, G.M. Mkoji and E.S. Loker, 2003. A newly-identified lineage of Schistosoma. Int. J. Parasitol., 33: 977-985.

Page, R.D.M., 1996. TreeView: An application to display phylogenetic trees on personal computers. Comput. Appl. Biosci., 12: 357-358.

Sato, Y., T.H. Le, R. Hiraike, M. Yukawa, T. Sakai, R.P.V.J. Rajapakse and T. Agatsuma, 2008. Mitochondrial DNA sequence and gene order of the Sri Lankan Schistosoma nasale is affiliated to the African/Indian group. Parasitol. Int., 57: 460-464.

Singh, A., A. Singh and S.S. Chaudhri, 2004. Visceral schistosomiasis of domestic animals in India: Humoral immune status of infected cattle, sheep and goats against major polypeptide antigens of Schistosoma indicum and $S$. spindale. Parasite Immunol., 26: 167-175.

Sneath, P.H.A. andR.R. Sokal, 1973. Numerical Taxonomy: The Principles and Practice of Numerical Classification. W.H. Freeman and Co., San Francisco.

Tamura, K., J. Dudley, M. Nei and S. Kumar, 2007. MEGA4: Molecular Evolutionary Genetics Analysis (MEGA) software version 4.0. Mol. Biol. Evol., 24: $1596-1599$.

Taylor, M., 2008. Global trends in schistosomiasis control. Bull. World Health Organ., 86: 738-738. 
Thompson, J.D., T.J. Gibson, F. Plewniak, F. Jeanmougi and D.G. Higgins, 1997. The CLUSTAL_X windows interface: Flexible strategies for multiple sequence alignment aided by quality analysis tools. Nucleic Acids Res., 25: 4876-4882.

Vercruysse, J. and S. Gabriel, 2005. Immunity to schistosomiasis in animals: An update. Parasite Immunol., 27: 289-295.

Vercruysse, J., D. Rollinson, M. van Heerden and V.R. Southgate, 2003. On the longevity of Schistosoma curassoni. J. Helminthol., 77: 89-90.

Walker, T.K., A.J. Simpson and D. Rollinson, 1989. Differentiation of Schistosoma mansoni from $S$. rodhaini using cloned DNA probes. Parasitology, 98: 75-80.

Webster, B.L., V.R. Southgate and D.T.J. Littlewood, 2006. A revision of the interrelationships of Schistosoma including the recently described Schistosoma guineensis. Int. J. Parasitol., 36: 947-955.

Yu, X.C., G.L. Wu, Y.J. Zhang, Y.Q.Wu, C.L. Zhu, Z.H. Zhang and B.Y. Chen, 2000. Studies of the genetic polymorphism of Schistosoma japonicum (Chinese mainland strains). Yi Chuan Xue Bao., 27: 498-505.
Zhang, G.J., C.P. Qiu, D.C. Qiu, Z.S. Chang, Z.H. Qin and M.Y. Xia, 2001. Study on molecular phylogeny of Schistosoma sinensium based on nuclear ribosomal DNA. Zhongguo Ji Sheng Chong Xue Yu Ji Sheng Chong Bing Za Zhi, 19: 201-204.

Zhao, G.H., D. Blair, X.Y. Li, J. Li and R.Q.Lin et al., 2011. The ribosomal intergenic spacer (IGS) region in Schistosoma japonicum: Structure and comparisons with related species. Infect. Genet. Evol., 11: 610-617.

Zhao, G.H., J. Li, F.C. Zou, X.H. Mo and Z.G. Yuan et al., 2009b. ISSR, an effective molecular approach for studying genetic variability among Schistosoma japonicum isolates from different provinces in mainland China. Infect. Genet. Evol., 9: 903-907.

Zhao, G.H., X.H. Mo, F.C. Zou, J. Li and Y.B. Weng et al., 2009a. Heterogeneity of class I and class II MHC sequences in Schistosoma japonicum from different endemic regions in mainland China. Parasitol. Res., 106: 201-206.

Zhou, X.N., L.Y. Wang, M.G. Chen, X.H. Wu and Q.W. Jiang et al., 2005. The public health significance and control of schistosomiasis in China-then and now. Acta. Trop., 96: 97-105. 